\title{
Oui le 18 mai à l'arrêté fédéral sur les soins médicaux de base
}

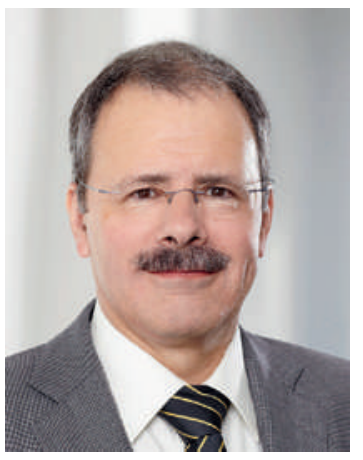

Le premier avril est cette année encore une date importante pour les 7500 médecins de famille et pédiatres de Suisse, celle du début d'une phase brûlante de la campagne de votation pour le nouvel article constitutionnel 117a. En effet, l'approbation par le peuple le 18 mai prochain de l'inscription de la médecine de premier recours et de la médecine de famille dans la Constitution fédérale signerait momentanément l'apogée d'une remarquable histoire à succès.

La persévérance des médecins de famille et des pédiatres tout au long du chemin qu'ils ont parcouru ces dernières années force l'admiration. Un court rappel: le Département de l'intérieur déclenche leur engagement en 2006, lorsqu'il décide une baisse linéaire de la valeur du point tarifaire pour les laboratoires au cabinet médical. Le premier avril de cette même année, 10000 médecins et sympathisants manifestent sur la Place fédérale. Leurs revendications vont bien au-delà des problèmes liés aux laboratoires au cabinet et visent plus largement de meilleures conditions de travail, la volonté d'être consultés lors des prises de décision et la nécessité de s'attaquer aux problèmes de la relève médicale par des améliorations de la formation prégraduée et postgraduée.

\section{Les médecins de famille et les pédiatres saisissent la chance de pouvoir garantir l'inscription des soins médicaux de base au plus haut niveau de la législation.}

L'enthousiasme créé par ce coup d'envoi impressionnant et son renforcement par le soutien de l'opinion publique ont assurément aidé les médecins de famille et les pédiatres à traverser les hauts et les bas de la vie politique au cours des années qui ont suivi. L'idée de vouloir se regrouper au sein de l'association nationale «Médecins de famille Suisse» fait progressivement son chemin et se révèle pertinente. En effet, pas plus tard qu'en 2008, la révision de la liste des analyses mandatée par le Conseil fédéral montre le peu de sérieux accordé à la revalorisation de la médecine de famille. Et malgré une résistance farouche, une nouvelle réduction des tarifs des laboratoires est décidée en 2009.

Paradoxalement, ce revers a donné un élan supplémentaire au projet de lancement de l'initiative populaire «Oui à la médecine de famille», qui s'est révélé être un véritable succès. Le seuil des 100000 signatures nécessaires est atteint en
76 jours. Lorsque l'initiative est déposée, cinq mois plus tard le premier avril 2010 -, 202256 signatures validées ont été récoltées. Avec l'approbation du contre-projet direct de la Commission du Conseil des Etats, les médecins de famille et les pédiatres saisissent la chance de pouvoir garantir l'inscription des soins médicaux de base au plus haut niveau de la législation. De plus, le masterplan «médecine de famille», découlant directement de l'initiative, reprend les points les plus importants défendus par les médecins de famille et les pédiatres, et de premiers succès ont déjà été enregistrés.

\section{Parler d'une seule voix et agir de manière proactive sont des facteurs de succès décisifs.}

Que retenir de ces événements? D'une part, qu'il est encourageant de voir que la patience et la ténacité peuvent payer en politique professionnelle, et d'autre part, que la proactivité et le courage dont ont fait preuve les médecins de famille et les pédiatres avec leur initiative populaire méritent le respect.

Mais avant tout, leur engagement montre l'ampleur de ce qui peut être atteint par l'union des forces en présence: de la grande manifestation au comité d'initiative en passant par le processus parlementaire, plusieurs organisations ont tiré sur la même corde et dans la même direction.

Par ailleurs, cela met aussi en évidence la puissance politique que nous, corps médical, risquerions de perdre en nous laissant monter les uns contre les autres et en nous égarant dans des intérêts particuliers. L'intervention du Conseil fédéral dans les tarifs, assortie d'une redistribution arbitraire de 200 millions de francs, constitue un terrain parfait pour provoquer des conflits entre spécialistes et médecins de premier recours. Mais précisément, lorsque nous condamnons avec véhémence cette intervention tarifaire, nous ne devons pas nous tromper de cible mais bien orienter nos critiques directement à l'encontre de la politique fédérale. En parallèle, nous devons nous concentrer sur nos forces et nos objectifs communs, en particulier pour donner corps à une solution réaliste, élaborée par les partenaires tarifaires sur la base de la révision globale du TARMED.

En octobre 2013, la Chambre médicale s'est déclarée solidaire avec les points défendus par les médecins de famille et les pédiatres et elle a soutenu l'article constitutionnel par une nette majorité de $74 \%$. Dans ce contexte, je vous appelle tous, mes chers membres de la FMH, à approuver le nouvel article constitutionnel sur les soins médicaux de base le 18 mai 2014.

Dr Jürg Schlup, président de la FMH 